PROCEEDINGS OF THE

AMERICAN MATHEMATICAL SOCIETY

Volume 138, Number 11, November 2010, Pages 4103-4107

S 0002-9939(2010)10445-8

Article electronically published on May 27, 2010

\title{
A NOTE ON THE YAMABE CONSTANT OF AN OUTERMOST MINIMAL HYPERSURFACE
}

\author{
FERNANDO SCHWARTZ
}

(Communicated by Richard A. Wentworth)

\begin{abstract}
Using an elementary argument we find an upper bound on the Yamabe constant of the outermost minimal hypersurface of an asymptotically flat manifold with nonnegative scalar curvature that satisfies the Riemannian Penrose Inequality. Provided the manifold satisfies the Riemannian Penrose Inequality with rigidity, we show that equality holds in the inequality if and only if the manifold is the Riemannian Schwarzschild manifold.
\end{abstract}

\section{INTRODUCTION AND MAIN RESULT}

A classic result by Hawking [7] states that each connected component of the outermost trapped surface in a spacelike slice of a spacetime that satisfies the dominant energy condition has to be, topologically, either toroidal or spherical. Hawking's result was recently extended to higher dimensions by Galloway and Schoen [6], where it is proved that, except for exceptional cases, the outermost trapped surfaces in any spacelike slice of a spacetime satisfying the dominant energy condition is of positive Yamabe type, i.e. admits a conformally related metric with positive scalar curvature. The exceptional cases were later ruled out by Galloway in 4 .

Inside the class of asymptotically flat manifolds with nonnegative scalar curvature $\mathscr{M}_{A F}^{+}$(which are usually interpreted as time-symmetric slices of spacetimes satisfying the dominant energy condition) the above results read as follows: the Yamabe constant (Euler characteristic, in dimension 3) of the outermost minimal hypersurface of a manifold in $\mathscr{M}_{A F}^{+}$is strictly positive.

In this paper we give an upper bound for the Yamabe constant (amount of components, in dimension 3 ) of the outermost minimal hypersurface inside a manifold in $\mathscr{M}_{A F}^{+}$. We prove that the inequality is rigid whenever the manifold satisfies the Riemannian Penrose Inequality with rigidity. (See Definition 3 below.) More precisely, we prove the following.

Theorem 1. Let $n \geq 3$ and consider $\left(M^{n}, g\right) \in \mathscr{M}_{A F}^{+}$containing an outermost minimal hypersurface $\Sigma$. The following holds:

(i) If $n=3$, then

$$
\#\{\text { components of } \Sigma\} \leq \frac{1}{2 \pi} m^{2} \omega_{2}\left(\left\|R^{M}\right\|_{L^{\infty}(\Sigma)}+2\|\operatorname{Ric}(\nu)\|_{L^{\infty}(\Sigma)}\right) .
$$

Received by the editors November 5, 2009 and, in revised form, February 8, 2010.

2010 Mathematics Subject Classification. Primary 53C21, 83C99.

This work was partially supported by The Leverhulme Trust.

(C)2010 American Mathematical Society Reverts to public domain 28 years from publication 
Equality is attained if and only if the part of $\left(M^{3}, g\right)$ outside the outermost minimal hypersurface is isometric to the Riemannian Schwarzschild 3-manifold of mass $m$. (See Definition 2.)

(ii) If $\left(M^{n}, g\right)$ satisfies the Riemannian Penrose Inequality (e.g. if $4 \leq n \leq 7$; cf. [2]), then

$Y\left(\Sigma,\left.g\right|_{\Sigma}\right) \leq(2 m)^{\frac{2}{n-2}}\left(\omega_{n-1}\right)^{\frac{2}{n-1}}\left(\left\|R^{M}\right\|_{L^{\infty}(\Sigma)}+2\|\operatorname{Ric}(\nu)\|_{L^{\infty}(\Sigma)}\right)$.

If, further, $(M, g)$ satisfies the Riemannian Penrose Inequality with rigidity (e.g. if $4 \leq n \leq 7$ and $\left(M^{n}, g\right)$ is spin; cf. [2]), then equality is attained if and only if the part of $\left(M^{n}, g\right)$ outside the outermost minimal hypersurface is isometric to the Riemannian Schwarzschild n-manifold of mass m.

We would like to point out that Galloway and Cai have an upper bound for the Yamabe constant of the boundary horizon whenever it has negative $\sigma$-invariant. (See Theorem 5 of [5].) Also, Theorem 2.3 of Corvino's paper [3] is similar, at least in spirit, to part $(i)$ of Theorem 1 above.

\section{Proof of Theorem 1}

Definition 2. For $n \geq 3$ and $m>0$ we define the Riemannian Schwarzschild $n$-manifold of mass $m$ to be the manifold $\left(\mathscr{S}_{n, m}, g_{n, m}\right):=\left(\mathbb{R}^{n} \backslash B_{r_{m}}(0),(1+\right.$ $\left.\left.\frac{m}{2} r^{2-n}\right)^{\frac{4}{n-2}} \delta_{i j}\right)$, where $r_{m}=\left(\frac{m}{2}\right)^{\frac{1}{n-2}}$ is the so-called Schwarzschild radius.

The Riemannian Schwarzschild manifold is the canonical model for a manifold in $\mathscr{M}_{A F}^{+}$that contains an outermost minimal surface. Indeed, it is well known that $\left(\mathscr{S}_{n, m}, g_{n, m}\right)$ is asymptotically flat and scalar flat, its boundary is a totally geodesic (thus minimal) $(n-1)$-sphere, and it does not contain any other compact minimal hypersurfaces. It is also known that the metric on its boundary is a positive constant multiple of the round metric on $S^{n-1}$.

Definition 3. An $n$-dimensional Riemannian manifold $(M, g)$ in $\mathscr{M}_{A F}^{+}$containing an outermost (compact) minimal hypersurface $\Sigma$ is said to satisfy the Riemannian Penrose Inequality if

$$
m \geq \frac{1}{2}\left(\frac{|\Sigma|}{\omega_{n-1}}\right)^{\frac{n-2}{n-1}}
$$

where $m$ is the ADM-mass of $(M, g)$, and $|\Sigma|$ denotes the area of $\Sigma$ with respect to $g$. We say that $(M, g)$ satisfies the Riemannian Penrose Inequality with rigidity $(\mathrm{RPI}+\mathrm{R})$ if it satisfies equation RPI and equality in RPI is attained if and only if the part of $(M, g)$ outside the outermost minimal hypersurface is isometric to the Riemannian Schwarzschild $n$-manifold of mass $m$.

What is currently known with respect to which manifolds satisfy the RPI and $\mathrm{RPI}+\mathrm{R}$ comes from a theorem of Bray and its generalization by Bray and Lee 1

Theorem 4 (Bray [1, Bray-Lee [2]). In dimension 3, any manifold in $\mathscr{M}_{A F}^{+}$containing an outermost minimal surface satisfies the $R P I+R$. In dimensions $4 \leq n \leq$ 7, any manifold in $\mathscr{M}_{A F}^{+}$containing outermost minimal hypersurfaces satisfies inequality RPI. Furthermore, if the manifold is spin, then $R P I+R$ holds.

\footnotetext{
${ }^{1}$ Huisken and Ilmanen also proved the 3-dimensional case of Theorem 4 in 8 . Nevertheless, their proof requires the outermost minimal surface to be connected.
} 
Remark. We use the Hawking-Galloway-Schoen Theorem in our proof to prove the rigidity statements. More precisely, we will use their stability result, which gives that $\chi\left(\Sigma^{2}\right)>0$ in dimension 3 , and $Y\left(\Sigma,\left.g\right|_{\Sigma}\right)>0$ in dimensions 4 and above, where $\Sigma$ is the outermost minimal hypersurface.

Proof of Theorem 1. To prove $(i)$, recall that the scalar curvature of a surface is twice its Gaussian curvature; thus

$$
8 \pi \#\{\text { components of } \Sigma\}=4 \pi \chi(\Sigma)=\int_{\Sigma} 2 K d A=\int_{\Sigma} R^{\Sigma} d A,
$$

where the first equality above follows from the Hawking-Galloway-Schoen Theorem, which gives $\chi\left(\Sigma^{2}\right)>0$. Using the Gauss equation we get

$$
=\int_{\Sigma}\left(R^{M}-2 \operatorname{Ric}(\nu)-|A|^{2}+H^{2}\right) d A,
$$

and by minimality $H=0$, so

$$
\leq|\Sigma|\left(\left\|R^{M}\right\|_{L^{\infty}(\Sigma)}+2|| \operatorname{Ric}(\nu) \|_{L^{\infty}(\Sigma)}\right) .
$$

Bray's result [1] gives that $(M, g)$ satisfies the RPI, therefore

$$
\leq 4 m^{2} \omega_{2}\left(\left\|R^{M}\right\|_{L^{\infty}(\Sigma)}+2\|\operatorname{Ric}(\nu)\|_{L^{\infty}(\Sigma)}\right) .
$$

If equality holds throughout in the above, then we can divide by the term $\left(\left\|R^{M}\right\|_{L^{\infty}(\Sigma)}+2\|\operatorname{Ric}(\nu)\|_{L^{\infty}(\Sigma)}\right)$ on both sides of the last inequality. (Note that $\left(\left\|R^{M}\right\|_{L^{\infty}(\Sigma)}+2\|\operatorname{Ric}(\nu)\|_{L^{\infty}(\Sigma)}\right)>0$ because $\Sigma$ has at least one component.) We recover the rigidity statement of the RPI in dimension 3, which holds because of Bray's result [1. Thus, the part of $\left(M^{3}, g\right)$ outside the outermost minimal hypersurface is isometric to the Riemannian Schwarzschild 3-manifold of mass $m$.

Conversely, consider the Riemannian Schwarzschild 3-manifold of mass $m$ (which clearly satisfies RPI+R). It is well known that this manifold is scalar flat, $\operatorname{Ric}(\nu)$ is a negative constant along $\Sigma$, and $\Sigma$ is totally geodesic. This way, the first inequality above is an equality. A straightforward calculation gives that RP] is an equality in this case, so the second inequality above is also an equality. This finishes the proof of $(i)$.

The proof of (ii) goes along similar lines. Indeed, taking into consideration that

$$
Y\left(\Sigma,\left.g\right|_{\Sigma}\right)=\inf \left\{\frac{\int_{\Sigma} R_{h}^{\Sigma} d V_{h}}{\left(\int_{\Sigma} d V_{h}\right)^{\frac{n-3}{n-1}}}: h \in\left[\left.g\right|_{\Sigma}\right]\right\} \leq \frac{\int_{\Sigma} R^{\Sigma} d V_{h}}{|\Sigma|^{\frac{n-3}{n-1}}}
$$

and using the Gauss equation and $H=0$, this gives

$$
\leq|\Sigma|^{1-\frac{n-3}{n-1}}\left(\left\|R^{M}\right\|_{L^{\infty}(\Sigma)}+2\|\operatorname{Ric}(\nu)\|_{L^{\infty}(\Sigma)}\right) .
$$

Combining this with the RPI (which holds by hypothesis) we obtain

$$
\leq(2 m)^{\frac{2}{n-2}}\left(\omega_{n-1}\right)^{\frac{2}{n-1}}\left(\left\|R^{M}\right\|_{L^{\infty}(\Sigma)}+2\|\operatorname{Ric}(\nu)\|_{L^{\infty}(\Sigma)}\right) .
$$

Now suppose that the above inequalities are all equalities. Hawking-GallowaySchoen gives that the term $\left(\left\|R^{M}\right\|_{L^{\infty}(\Sigma)}+2\|\operatorname{Ric}(\nu)\|_{L^{\infty}(\Sigma)}\right)$ is positive, so we may divide by it on both sides of the last inequality. We obtain the equality case of the RPI. If $(M, g)$ satisfies the RPI+R, the statement follows. 
We now show that these are all equalities for the Schwarzschild manifolds $\left(\mathscr{S}_{n, m}\right.$, $\left.g_{n, m}\right), n \geq 4$. The first inequality above is actually an equality because of the solution to the Yamabe problem. Indeed, it is well known that the round metric on a sphere (and therefore any positive multiple of it, because of scaling) minimizes the above quotient. (See e.g. 9.) Since the metric on the boundary of $\mathscr{S}_{n, m}$ is a multiple of the round metric on the $(n-1)$-sphere (because the conformal factor $\left(1+\frac{m}{2} r^{2-n}\right)^{\frac{4}{n-2}}$ is constant on the boundary), it follows that $Y\left(\Sigma,\left.g\right|_{\Sigma}\right)=\frac{\int_{\Sigma} R^{\Sigma} d V_{h}}{|\Sigma|^{\frac{n-3}{n-1}}}$.

To finish the proof we note that $g_{n, m}$ is scalar flat, $\operatorname{Ric}(\nu)$ is a negative constant along $\Sigma$, and $\Sigma$ is totally geodesic. This way, the second inequality above is an equality. A straightforward calculation gives that [RPI is an equality in this case, so the third inequality above is also an equality.

Remark. It is worth noting that along the way we have shown that

$$
|\Sigma| \geq\left(\frac{Y\left(\Sigma,\left.g\right|_{\Sigma}\right)}{\left(\left\|R^{M}\right\|_{L^{\infty}(\Sigma)}+2|| \operatorname{Ric}(\nu) \|_{L^{\infty}(\Sigma)}\right)}\right)^{(n-1) / 2}
$$

which is of interest in itself and may be compared with equation (16) of [5].

\section{OpEN PROBLEM}

It would be very interesting to determine in which cases the upper bound we find in Theorem 1 is an improvement over the Yamabe constant of the round sphere, which is a known upper bound for $Y\left(\Sigma,\left.g\right|_{\Sigma}\right)$.

\section{ACKNOWLEDGEMENTS}

This work was carried out during a visit to the Warwick Mathematics Institute. The author would like to thank Peter Topping and Mario Micallef for their hospitality. The author also would like to thank the referee for useful comments.

\section{REFERENCES}

[1] Hubert L. Bray, Proof of the Riemannian Penrose inequality using the positive mass theorem, J. Differential Geom. 59 (2001), no. 2, 177-267. MR1908823 (2004j:53046)

[2] Hubert L. Bray and Dan A. Lee, On the Riemannian Penrose inequality in dimensions less than eight, Duke Math. J. 148 (2009), no. 1, 81-106. MR2515101

[3] Justin Corvino, A note on asymptotically flat metrics on $\mathbb{R}^{3}$ which are scalar-flat and admit minimal spheres, Proc. Amer. Math. Soc. 133 (2005), no. 12, 3669-3678 (electronic), DOI 10.1090/S0002-9939-05-07926-8. MR2163606 (2007a:53077)

[4] Gregory J. Galloway, Rigidity of marginally trapped surfaces and the topology of black holes, Comm. Anal. Geom. 16 (2008), no. 1, 217-229. MR2411473 (2009e:53087)

[5] Mingliang Cai and Gregory J. Galloway, On the topology and area of higher-dimensional black holes, Classical Quantum Gravity 18 (2001), no. 14, 2707-2718, DOI 10.1088/02649381/18/14/308. MR:1846368(2002k:83051)

[6] Gregory J. Galloway and Richard Schoen, A generalization of Hawking's black hole topology theorem to higher dimensions, Comm. Math. Phys. 266 (2006), no. 2, 571-576. MR2238889 (2007i:53078)

[7] S. W. Hawking, Black holes in general relativity, Comm. Math. Phys. 25 (1972), 152-166. MR0293962 (45:3037) 
[8] Gerhard Huisken and Tom Ilmanen, The inverse mean curvature flow and the Riemannian Penrose inequality, J. Differential Geom. 59 (2001), no. 3, 353-437. MR1916951|(2003h:53091)

[9] Richard Schoen, Conformal deformation of a Riemannian metric to constant scalar curvature, J. Differential Geom. 20 (1984), no. 2, 479-495. MR788292(86i:58137)

Department of Mathematics, University of Tennessee, Knoxville, Tennessee 37996 0614

E-mail address: fernando@math.utk.edu 